\title{
Avaliação do efeito antiparasitário de probióticos em primatas do velho mundo, mantidos em cativeiro, no Zoológico Municipal de Volta Redonda - RJ
}

\author{
Evaluation of the effect of antiparasitic probiotics in the old world \\ primates kept in captivity in Municipal Zoo Volta Redonda - RJ
}

\author{
Carlos Alberto Sanches Pereira ${ }^{1}$ \\ Mayara de Souza Costa ${ }^{2}$ \\ 1 Docente do Centro Universitário de Volta Redonda, Departamento de Ciências Biológicas - UniFOA. \\ 2 Discente do curso de Ciências Biológicas do Centro Universitário de Volta Redonda - UniFOA.
}

\section{RESUMO}

As doenças em animais de cativeiro vêm apresentando um aumento expressivo, principalmente as relacionadas ao manejo inadequado, representando um ambiente propício à sua disseminação. Em consequência desses fatores, há muitos distúrbios na microbiota normal do animal, alterando o equilíbrio intestinal. Corroborando para as desvantagens ligadas ao efeito antiparasitário de vermífugos e antibióticos, atualmente, os probióticos são consideradas ferramentas alternativas no tratamento e na prevenção das diarreias, pois contêm microorganismos benéficos que ajudam a suprimir os patógenos. Analisando-se o prisma das vantagens que os probióticos proporcionam, o presente trabalho teve como objetivo a avaliação do efeito antiparasitário de probióticos à base de lactobacilos, em primatas do velho mundo, mantidos em cativeiro, no Zoológico Municipal de Volta Redonda, RJ. Os primatas Macaco rhesus, Babuíno sagrado e Babuíno sagrado fêmea receberam, diariamente, $10 \mathrm{ml}$ de preparado com $10^{9} \mathrm{UFC} / \mathrm{mL}$ do Lactobacillus casei ATCC 7469, Lactobcillus plantarum ATCC 8014, Lactobacillus fermentum ATCC 9338 e Lactobacillus acidophilus ATCC 7469 na forma de "pool", em condições assépticas, num período de 135 dias, sendo administrado junto ao pão com mel, para melhoria do sabor, 30 minutos antes da dieta diária de cada animal.

\section{Palavras-chave}

Probióticos, Lactobacillus, Trichuris trichiura.

\begin{abstract}
The illnesses with captivity animals presents an expressive increase, mainly related to the inadequate handling, representing a propitious environment to the dissemination of illnesses. The consequences of these factors in the intestinal balance has many riots in normal microbiota of the animal, leading to serious parasitic illnesses. Corroborating on disadvantages to the antiparasitic effects of vermifuges and antibiotics, the probiotics are considered an alternative tool in the treatment and the prevention of diarrheas, therefore it contains beneficial microorganisms that help to suppress the pathogenic ones. Analyzing the advantages that the probiotics provide, the present work has as objective the evaluation of the antiparasitic effect of probiotics based on lactobacillus in primates of the old world in captivity in the municipal Zoo in Volta Redonda/RJ. The animals had daily received $10 \mathrm{ml}$ from a chemical preparation with $10 \mathrm{UFC} / \mathrm{mL}$ of Lactobacillus casei ATCC 7469, Lactobcillus plantarum ATCC 8014, Lactobacillus fermentum ATCC 9338 e Lactobacillus acidophilus ATCC 7469, in aseptic conditions, being managed with bread and honey for better flavor.
\end{abstract}

\section{Keywords}

Probiotics, Lactobacillus, Trichuris trichiura.

\section{Como você deve citar?}

PEREIRA, Carlos Alberto Sanches; COSTA, Mayara de Souza. Avaliação do efeito antiparasitário de probióticos em primatas do velho mundo, mantidos em cativeiro, no Zoológico Municipal de Volta Redonda - RJ. Cadernos UniFOA, Volta Redonda, n. 26, p. 123-129, dez. 2014. 
Avaliação do efeito antiparasitário de probióticos em primatas do velho mundo, mantidos em cativeiro, no Zoológico Municipal de Volta Redonda - RJ

\section{INTRODUÇÃO}

As doenças com animais de cativeiro vêm apresentando um aumento expressivo, principalmente as relacionadas ao manejo inadequado, à alimentação, ao estresse, às condições climáticas e à troca constante de habitats. Além disso, espécies selvagens, vivendo em condições diferentes do seu habitat natural, tornam-se mais vulneráveis à contaminação e disseminação de doenças.

A infecção e a disseminação de patógenos podem ocorrer entre os animais mantidos no próprio zoológico, para os animais livres, funcionários e ao público visitante (SILVA et al., 2008). Como consequência desse panorama, ocorrem distúrbios na microbiota normal do animal, alterando o equilíbrio intestinal, o que pode ocasionar doenças parasitárias graves.

A utilização de antibióticos ou vermífugos é considerada de grande importância no crescimento e na produtividade do animal. Em contrapartida, o uso desses antimicrobianos nas dietas de animais foi questionado em vários países. A razão está relacionada com a formação de um "estoque" de bactérias entéricas resistentes a drogas e capazes de transferir a resistência para os descendentes. Desse modo, tornou-se crescente a proibição do uso dos antimicrobianos (FREITAS, 2008).

Portanto, contribuindo para as desvantagens ligadas ao efeito antiparasitário, a necessidade de tratamentos repetitivos e a elevada reinfecção dos animais, têm-se estimulado a procura de novas terapias, promovendo uma ferramenta alternativa no controle das infecções parasitárias (FUJIWARA et al., 2007). Nesse sentido, os probióticos são considerados boas ferramentas para o tratamento, pois são micro-organismos que, quando administrados em doses adequadas, promovem o bem estar dos animais, contribuindo na redução da carga parasitária, na manutenção da microbiota intestinal e na colonização de bactérias benéficas. Com isso, diminuem a colonização de micro-organismos indesejáveis, auxiliando na prevenção e no tratamento de alterações intestinais (WHO, 2003).

O efeito antiparasitário dos probióticos, nos estudos de Pereira (2007), mostrou ser efetivo no tratamento de Eimeriose, interrompendo a eliminação de oocistos de Eimeria em ratos que foram contaminados e, posteriormente, tratados com probiótico e vice-versa, demonstrando ganho de peso, melhor conversão alimentar e aumento do número de neutrófilos segmentados.

Segundo os resultados de Coêlho (2010), a preparação probiótica composta de Lactobacillus acidophilus, Lactobacillus plantarum e Lactobacillus delbruekii, na forma de "pool", apresentaram potencial para a diminuição de ovos por grama de fezes em cães naturalmente infectados por Ancilostoma spp. de 88,83\%, ressaltando-se que, após cessar o tratamento, esses valores aumentaram, além de induzir uma ativação na resposta imune, melhorando o número de eritrócitos, concentração de hemoglobina e aumento do hematócrito.

A preparação probiótica administrada por Teixeira (2011) apresentou eficácia sobre Haemonchus contortus em ovinos, quando comparada à administração de anti-helmíntico, em que o "pool" de Lactobacillus estimulou o sistema imune dos animais, além de apresentarem melhores condições fisiológicas.

Tais estudos suplementam a relevância de pesquisas descrita por Souza et al. (2010), em que os primatas são particularmente vulneráveis aos efeitos do parasitismo, por viverem em grupos sociais estreitos, o que facilita a transmissão dos parasitos que, no caso de Trichuris, ocorre pelo hábito da ingestão fecal e consequente ingestão de ovos.

O Trichuris é um nematódeo, no qual o verme adulto é facilmente identificado pela extremidade anterior afilada. Ocasiona, ao hospedeiro, uma anemia severa em grandes infestações e atraso no desenvolvimento físico do animal, tendo maior prevalência em regiões quentes e úmidas (HUGGINS; MEDEIROS, 2001). 
O presente trabalho teve como objetivo avaliar o efeito antiparasitário de um preparado probiótico constituído por um "pool" de Lactobacillus, em primatas do velho mundo, naturalmente, infectados com Trichuris trichiura.

\section{MATERIAL E MÉTODOS}

O presente trabalho foi desenvolvido no laboratório de biotecnologia do Centro Universitário de Volta Redonda - UniFOA e sua fase de implantação foi realizada no Zoológico Municipal de Volta Redonda, RJ.

Os animais estudados foram Macaco rhesus (Macaca mulatta), Babuíno sagrado (Papio hamadryas) e Babuíno sagrado (Fêmea) (Papio hamadryas), os quais vivem em recintos separados.

A preparação probiótica avaliada no presente trabalho foi constituída de um "pool" de Lactobacillus, composto pelas seguintes espécies: Lactobacillus casei ATCC 7469, Lactobacillus plantarum ATCC 8014, Lactobacillus fermentum ATCC 9338 e Lactobacillus acidophilus ATCC 7469, que foram, gentilmente, cedidos pelo Laboratório de Probióticos da Escola de Engenharia de Lorena / USP.

As culturas foram mantidas sob congelamento a $-20^{\circ} \mathrm{C}$ em criotubos, contendo caldo MRS com glicerol a $40 \%$ estéril, com o intuito de armazená-los para posteriores ativações. Para a ativação dos micro-organismos, as amostras foram descongeladas até que atingissem a temperatura ambiente e ativadas por três vezes consecutivas em tubos, contendo $2 \mathrm{~mL}$ de caldo MRS (Man Rogosa Sarpe) estéril e incubadas a $37^{\circ} \mathrm{C}$, por um período de 24 horas. Após a última ativação, os $2 \mathrm{~mL}$ da amostra crescida foram transferidos para garrafas de vidro, contendo $70 \mathrm{~mL}$ de caldo MRS esterilizados a $121^{\circ} \mathrm{C}$ por 15 minutos, as quais, em sequência, foram incubadas a $37^{\circ} \mathrm{C}$, por um período de 24 horas. Após esse procedimento, as amostras foram colocadas em um único recipiente estéril, com o objetivo de obter o "pool", constituído, aproximadamente, por $10^{9} \mathrm{UFC} / \mathrm{mL}$ de Lactobacillus, para posteriores administrações aos primatas. Esse "pool" foi armazenado à temperatura de $5^{\circ} \mathrm{C}$, durante os sete dias de administração do preparado probiótico aos primatas, de acordo com a metodologia de Pereira (2007).

Para a administração do preparado probiótico, os animais receberam, diariamente, $10 \mathrm{~mL}$, sendo administrado junto ao pão com mel. A administração foi realizada 30 minutos antes da dieta diária dos animais com ajuda do tratador do zoológico, de forma a obter uma ingestão segura do preparado. 0 probiótico foi aplicado durante 135 dias.

Em relação à coleta de fezes, um dia antes, foi realizada uma limpeza total dos recintos ocupados pelos primatas, utilizando-se a vassoura de fogo, cuja função é esterilizar toda área onde possa conter carrapatos ou outros parasitas.

As fezes foram coletadas dos recintos em potes estéreis, com volume de 4 gramas a cada 15 dias e transportadas para o laboratório de Biotecnologia. No laboratório, as amostras foram armazenadas em ambiente refrigerado até serem realizados os exames parasitológicos, durante o período máximo de 48 horas. Para as amostras do teste qualitativo e quantitativo foram avaliadas três amostras.

Para a primeira análise parasitológica foi realizado o teste qualitativo, utilizando-se a técnica de Sheather Centrífugo flutuação, que tem o intuito de identificar e classificar os parasitos intestinais. A técnica fundamenta-se no princípio da diferença de densidade específica entre os ovos de helmintos, cistos e oocistos de protozoários e o material fecal (MONTEIRO, 2011). 
Avaliação do efeito antiparasitário de probióticos em primatas do velho mundo, mantidos em cativeiro, no Zoológico Municipal de Volta Redonda - RJ

Num primeiro momento, foi realizada a maceração e a homogeneização das fezes com a solução de sacarose, na qual foram passadas no parasito filtro e transferidas para o tubo de ensaio. Em seguida, as amostras foram centrifugadas por 2 minutos e retirados $10 \mu \mathrm{l}$ da suspensão fecal, para exame em lâmina. Foi adicionada uma solução de lugol para melhor visualização dos parasitos.

Para a análise quantitativa foi utilizada a técnica McMaster, desenvolvida por Gordon e Whitlock (1939), que consiste em identificar e quantificar a presença de ovos e oocistos em amostras de fezes.

Em 2 gramas de fezes, acrescentou-se $28 \mathrm{ml}$ de solução hipersaturada de cloreto de sódio; homogeneizou-se bem a suspensão fecal, passando-a através de peneira; centrifugou-se por 2 minutos e com uma pipeta volumétrica de Pasteur; retirou-se uma pequena amostra, preenchendo as duas áreas da câmara de McMaster; deixou-se em descanso por cinco minutos e logo observou-se no microscópio óptico Time-in, em aumento de 10x, fazendo a contagem dos ovos ou oocistos encontrados em ambas as áreas, focando sempre as linhas das retículas, que foi inteiramente coberta.

0 valor total de ovos encontrados na área esquerda mais o total de ovos da área direita, multiplicados por 50, representam o total de ovos encontrados por grama de fezes.

\subsection{Análise Estatística}

Os resultados foram analisados, utilizando-se o software Excel para verificar a eficácia do probiótico em cada animal, em cada coleta, e também em todo o tratamento, correlacionando-os entre a contagem de OPG de fezes.

Foi determinada a eficácia de cada tratamento, conforme metodologia proposta por Teixeira (2011), empregando-se a seguinte fórmula:

$$
\% \text { Eficácia }=\frac{\text { Média } O P G_{\text {dia anterior }}-\text { Média } O P G_{\text {dia final }}}{\text { Média } O P G_{\text {dia anterior }}} \times 100
$$

Fonte: do autor, 2014.

\section{RESULTADOS E DISCUSSÃo}

Os resultados encontrados com o teste qualitativo mostram que todos os primatas apresentam o mesmo nematóide, Trichuris trichiura, podendo estar relacionado ao fato de viverem, frequentemente, em grupos sociais estreitos ou com a troca constante de cativeiro entre os mesmos, que os torna mais vulneráveis aos efeitos do parasitismo, facilitando sua transmissão, conforme os estudos de Souza et al., (2010).

Assim, como os estudos realizados por Ximenes (1997), nos quais foram encontrados em Callithrix jacchus (saguis), endoparasitas, como os helmintos Trichuris trichiura e Oesophagostomum sp. e o protozoário Eimeria sp., enquanto que, o Saguinus fuscicollis weddelli apresentou Trichuris trichiura e o Saguinus imperator apresentou Trichostrongylus sp. 
De acordo com os resultados expressos na Tabela 1, pode-se observar que a partir do $1^{\circ}$ dia de tratamento, os 3 animais tiveram redução e eliminação de ovos do Trichuris trichiura em suas fezes, que permaneceram constante até o final do experimento entre os dois machos, o que não ocorreu com a Papio hamadryas (Fêmea) que, a partir da $2^{\circ}$ coleta, começou a aumentar a eliminação dos ovos nas fezes.

Tabela 1 - Resultados por grama de fezes do teste quantitativo (OPG).

\begin{tabular}{cccccccccccc}
\hline & \multicolumn{10}{c}{ Ovos por grama de fezes } \\
\hline Animais & \multicolumn{10}{c}{ Dias de Coleta } \\
\hline & $\mathbf{0 0}$ & $\mathbf{1 1}^{\circ}$ & $\mathbf{2 2}^{\circ}$ & $\mathbf{4 3}^{\circ}$ & $\mathbf{4 4}^{\circ}$ & $\mathbf{5 5 ^ { \circ }}$ & $\mathbf{6 6}^{\circ}$ & $\mathbf{7 7}^{\circ}$ & $\mathbf{T 8}^{\circ}$ & $\mathbf{9 9}^{\circ}$ \\
\hline Macaca mulatta & 1185 & 1166 & 1133 & 1116 & 1109 & 1100 & 1100 & 993 & 983 & 883 \\
\hline Papio hamadryas & 2250 & 1216 & 2200 & 2200 & 1183 & 1166 & 1150 & 1134 & 1134 & 1116 \\
\hline Papio hamadryas (Fêmea) & 3383 & 3350 & 3366 & 4400 & 4417 & 4433 & 4450 & 4466 & 4470 & 4483 \\
\hline
\end{tabular}

Fonte: do autor, 2014.

Nesse contexto, vale salientar que Teixeira (2011) observou que, na contagem de OPG no grupo de ovelhas em que foi administrado o probiótico, após 90 dias de experimento, manteve-se em níveis constantes, ressaltando que o "pool" de micro-organismos probióticos, apesar de não apresentar uma ação direta sobre Haemonchus contortus, foi capaz de manter o equilíbrio entre o parasito e hospedeiro.

Entretanto, o Papio hamadryas (Fêmea) obteve um aumento de OPG. A partir da primeira coleta, o mesmo apresentava uma ferida que resultou na amputaçãode sua cauda, podendo então ter influenciado negativamente nos benefícios trazidos pelo probiótico.

É notável que, decorridos 135 dias do início do experimento, a avaliação da eficácia do tratamento sobre o parasita Trichuris trichiura, demonstrado na Tabela 2, obteve uma redução de OPG, onde, o tratamento com o Macaca mulatta, em apenas 90 dias, não apresentou nenhuma redução e o Papio hamadryas, após 45 dias de tratamento, também não apresentou redução, porém após 15 dias de experimento, demonstrou redução máxima de 13,6\%.Tabela 2 - Valores de eficácia de redução de OPG nas fezes.

\begin{tabular}{cccccccccc}
\hline Animais & \multicolumn{10}{c}{ Tempo de experimento (dias) } \\
\hline & $\mathbf{1 5}$ & $\mathbf{3 0}$ & $\mathbf{4 5}$ & $\mathbf{6 0}$ & $\mathbf{7 5}$ & $\mathbf{9 0}$ & $\mathbf{1 0 5}$ & $\mathbf{1 2 0}$ & $\mathbf{1 3 5}$ \\
\hline Macaca mulatta & $10,3 \%$ & $19,9 \%$ & $12,8 \%$ & $6,0 \%$ & $8,2 \%$ & $0,0 \%$ & $7,0 \%$ & $10,7 \%$ & $0,0 \%$ \\
\hline Papio hamadryas & $13,6 \%$ & $7,4 \%$ & $0,0 \%$ & $8,5 \%$ & $9,3 \%$ & $9,6 \%$ & $10,7 \%$ & $0,0 \%$ & $13,4 \%$ \\
\hline Papio hamadryas (Fêmea) & $8,6 \%$ & $0,0 \%$ & $0,0 \%$ & $0,0 \%$ & $0,0 \%$ & $0,0 \%$ & $0,0 \%$ & $0,0 \%$ & $0,0 \%$ \\
\hline
\end{tabular}

Fonte: do autor, 2014.

Nesse sentido, vale ressaltar que o resultado de eficácia dos primatas Macaca mulatta e Papio hamadryas macho podem estar relacionados com a administração diária do preparado probiótico em pequenas doses (Pereira, 2007) e a limpeza dos recintos a cada 15 dias, contribuindo para a diminuição da infestação do parasito Trichuris trichiura.

Souza et al. (2010) encontraram infecções com Trypanoxyuris (Trypanoxyuris) minutus em Alouatta guariba clamitans, tendo como influência o confinamento dos animais, propiciando uma maior transmissão dos parasitos, tendo como consequências, os efeitos vulneráveis do parasitismo 
Avaliação do efeito antiparasitário de probióticos em primatas do velho mundo, mantidos em cativeiro, no Zoológico Municipal de Volta Redonda - RJ

por, frequentemente, viverem em grupos sociais estreitos, podendo explicar a ocorrência de estresse no Papio hamadryas fêmea e o aumento de parasitoses intestinais, devido à ferida em sua calda.

Os resultados apresentados na Tabela 3 demonstram que, ao final do tratamento, ou seja, após 135 dias, o tratamento com o Macaca mulatta obteve eficácia máxima, apresentando redução de 55,1\% e o Papio hamadryas apenas $1,5 \%$ a menos, observando-se uma eficácia no que se refere à redução parasitária, sugerindo que o tratamento alternativo com os probióticos beneficiou os primatas.

Tabela 3 - Valores de eficácia total.

\begin{tabular}{cc}
\hline Animais & Eficácia Total (\%) \\
\hline Macaca mulatta & $55,1 \%$ \\
\hline Papio hamadryas & $53,6 \%$ \\
\hline Papio hamadryas (Fêmea) & $0,0 \%$ \\
\hline
\end{tabular}

Fonte: do autor, 2014.

Resultado semelhante foi relatado por Teixeira (2011), que registrou efeitos positivos do uso de probióticos, frente às infecções parasitárias causadas por Haemonchus contortus em ovinos, nas quais, a preparação constituída por quatro cepas de Lactobacillus, na forma de "pool", apresentou uma eficácia máxima de $66,3 \%$ na redução do número de OPG, após 30 dias de tratamento.

Entretanto, o Papio hamadryas (Fêmea) não demonstrou nenhuma redução de OPG de fezes total. Somente nos primeiros 15 dias de tratamento observou-se uma redução de $8,6 \%$, destacando que a preparação probiótica não exerceu efeito sobre a prevenção da infestação de Trichuris trichiura.

Por outro lado, Coêlho (2010) demonstrou eficácia sobre o uso de probiótico no combate à ancilostomíase canina, utilizando o preparado probiótico composto de Lactobacillus acidophilus, $L$. delbruekii e L. plantarum, na forma de "pool", com uma redução de $88,83 \%$ do número de ovos por grama de fezes (OPG), ressaltando que, após cessar o tratamento, esses valores tenderam a aumentar, verificando-se, dessa forma, a necessidade da continuação do tratamento como rotina na dieta diária dos animais, como alternativa para mantê-los livres de parasitos.

\section{CONCLUSÃO}

Os resultados obtidos no presente trabalho permitem concluir que a preparação probiótica constituída de Lactobacillus, na forma de "pool", apresentou um potencial positivo no efeito antiparasitário nos primatas Macaca mulatta e Papio hamadryas, do velho mundo, mantidos no Zoológico Municipal de Volta Redonda, promovendo a redução do número de OPG nas fezes e uma eficácia, após 135 dias de tratamento.

\section{AGRADECIMENTOS}

Agradeço às técnicas de laboratório Renata Coutinho e Thais Boechat, pelo auxílio prestado e ao Zoológico Municipal de Volta Redonda - RJ, pela disponibilização dos animais para o estudo. 


\section{REFERÊNCIAS}

COELHO, M. D. G. Avaliação do uso de probiótico no combate a infecção causada por Ancylostomidae em cães naturalmente infectados. 2010. 94 p. Tese (Doutorado em Ciências) Escola de Engenharia de Lorena, Lorena, SP.

FREITAS, C. M. Efeito da administração de bactérias ácido-láticas sobe o ganho de peso de leitões na maternidade. 2008, 49 p. Dissertação (Mestrado em Medicina Veterinária). Universidade de São Paulo, SP.

FUJIWARA, R. T. et al. Reduction of worm fecundity and canine host lod loss mediates protection against hookworm infection elicited by vaccination with recombinant Ac-16. Clinical and Vaccine Immunology. v. 14, n. 3, p. 281-287, 2007.

GORDON, H. M. C. L.; WHITLOCK, A. V. A new technique for counting nematode eggs in sheep feces. Journal Council Scientific Industry Research. Australia, v. 12, p. 50-52, 1939.

HUGGINS D. W.; MEDEIROS L. B. Tricuríase: abordagem atual das doenças infecciosas e parasitárias. Huggins Medicin Tropic, v. 1, p. 309-316, 2001.

MONTEIRO, S. G. Parasitologia na medicina veterinária. São Paulo: Roca, 2011, 356 p.

PEREIRA, C. A. S. Avaliação do efeito do uso de probiótico sobre espécies do gênero Eimeria em Rattus norvegicus. 2007. 76 p. Tese (Doutor em Biotecnologia Industrial) Escola de Engenharia de Lorena, Lorena, SP.

SILVA A. S. et al. Ocorrência de protozoários gastrintestinais em primatas mantidos em cativeiro na região sul do Brasil. Ciência Rural. v. 38, n. 9, 2008.

SOUZA, D. P. et al. Ocorrência de Tryxyuris (Tryxyuris) minutus (Nematoda, Oxyuridae) em Alouatta guariba em Minas Gerais, Brasil. Revista Brasileira de Parasitologia Veterinária. v. 19, n. 2, p. 124-126, 2010.

TEIXEIRA, R. S. Avaliação do efeito de micro-organismo probióticos sobre Haemonchus contortus em ovinos. 2011. 67p. Dissertação (Mestrado em Ciências) Escola de Engenharia de Lorena, Lorena, SP.

WHO. International review panel evaluation of the termination of the use of antimicrobial growth promoters in Denmark. World Health Organization, v. 1, p. 6-9, 2003.

XIMENES, M. F. F. M. Parasitismo por helmintos e protozoários no sagüi-comum (Callithrix jacchus). In: A PRIMATOLOGIA NO BRASIL, 6, 1997, Natal. Anais... Natal: Sociedade Brasileira de Primatologia, 1997 , p. 249-256. 